\title{
Magnetically Induced Current Densities in Toroidal Carbon Nanotubes
}

\author{
Reiter, Kevin
}

2019-06-20

Reiter , K, Weigend , F , Wirz , L N , Dimitrova , M \& Sundholm , D 2019 , ' Magnetically Induced Current Densities in Toroidal Carbon Nanotubes ' , Journal of Physical Chemistry C , vol. 123 , no. 24 , pp. 15354-15365 . https://doi.org/10.1021/acs.jpcc.9b03769

http://hdl.handle.net/10138/304670

https://doi.org/10.1021/acs.jpcc.9b03769

cc_by

publishedVersion

Downloaded from Helda, University of Helsinki institutional repository.

This is an electronic reprint of the original article.

This reprint may differ from the original in pagination and typographic detail.

Please cite the original version. 


\title{
Magnetically Induced Current Densities in Toroidal Carbon Nanotubes
}

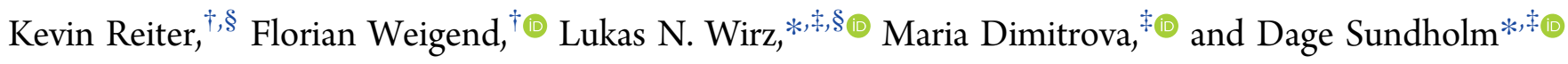 \\ ${ }^{\dagger}$ Institute of Nanotechnology, Karlsruhe Institute of Technology (KIT), Hermann-von-Helmholtz-Platz 1, D-76344 \\ Eggenstein-Leopoldshafen, Germany \\ ${ }^{\ddagger}$ Department of Chemistry, University of Helsinki, P.O. Box 55, A. I. Virtasen Aukio 1, FIN-00014 Helsinki, Finland
}

Supporting Information

\begin{abstract}
Molecular structures of toroidal carbon nanotubes (TCNTs) have been constructed and optimized at the density functional theory (DFT) level. The TCNT structures have been constrained by using point groups with high symmetry. TCNTs consisting of only hexagons (polyhex) with armchair, chiral, and zigzag structures as well as TCNTs with pentagons and heptagons have been studied. The employed method for constructing general polyhex TCNTs is discussed. Magnetically induced current densities have been calculated using the gauge-including magnetically induced currents (GIMIC) method. The strength of the magnetically induced ring currents has been obtained by integrating the current density passing a plane cutting the ring of the TCNT. The main pathways of the current density have been identified by visualizing the current density. The calculations show that the strength of the diatropic ring current of polyhex TCNTs with an armchair structure generally increases with the size of the TCNT, whereas TCNTs with a zigzag structure sustain very weak diatropic ring currents. Some of the TCNTs with pentagons and heptagons sustain a strong diatropic ring current, whereas other TCNT structures with pentagons and heptagons sustain paratropic ring currents that are, in most cases, relatively weak. We discuss the reasons for the different behaviors of the current density of the seemingly similar TCNTs.
\end{abstract}

\section{INTRODUCTION}

A torus is a topological object with genus 1 ; that is, colloquially speaking, it has one hole. The Gaussian curvature integrated over the surface of a torus is zero, implying that a cubic graph that can be embedded on the surface of a torus may consist of only faces with six sides, that is, hexagons. Alternatively, it may contain faces smaller than a hexagon, which have to be compensated by larger faces, for example, equal numbers of pentagons and heptagons, fulfilling the general topology relation in eq $1^{1,2}$

$$
\sum_{f}^{\text {faces }}\left(6-n_{f}\right)=12(1-g)
$$

where $n_{f}$ is the number of edges of the individual faces, and $g$ is the genus of the object. Structures of toroidal carbon nanotubes (TCNTs) that contain not only hexagons but also an equal number of pentagons and heptagons have been reported in refs 3, 13 and discussed in detail in ref 14. TCNTs have also been observed experimentally. ${ }^{15-18}$

The fascinating topology of the TCNTs might also lead to novel molecular magnetic properties, ${ }^{19,20}$ which means that studies of magnetically induced current densities in TCNTs are of interest. The magnetically induced current density is expected to flow mainly along the major ring of the torus when a magnetic field is applied perpendicular to the torus. In chiral TCNTs, the current density might, to some extent, follow the carbon network, leading to a helical structure of the current density. When that is the case, one of its vector components forms a current pathway around the minor ring perpendicular to the major ring. ${ }^{21,22}$ The vector component of the current density flowing around the minor ring would then induce a magnetic field along the major ring, leading to a magnetic anapole moment. ${ }^{7,19,20,23,24} \mathrm{~A}$ inhomogenious magnetic field is required for inducing an anapole moment in achiral tori, whereas a uniform field is sufficient for inducing an anapole moment in chiral tori. ${ }^{7}$ The diatropic ring current around the major ring gives rise to an induced magnetic field in the opposite direction to the applied magnetic field, whereas the induced magnetic moment has the same direction as the applied one when the induced ring current is paratropic.

Planar organic molecules fulfilling the $[4 n+2] \pi$-electron count rule are, in general, aromatic. ${ }^{25-33}$ The applicability of the aromaticity concept has been extended to complex

Received: April 22, 2019

Revised: $\quad$ May 17, 2019

Published: May 22, 2019 
molecular rings consisting of annelated or connected smaller rings. ${ }^{34-51}$ Even though the main molecular ring is nonplanar or even twisted, rings can still fulfill the generalized Hückel rule for aromaticity. ${ }^{37,52}$ The molecular aromaticity of complex structures such as TCNTs is not determined merely by the number of $\pi$ electrons of the conjugation pathway, but aromaticity may also be a topological property in a broader sense.

In this work, we investigate the magnetically induced current density of a number of TCNTs. We have studied TCNTs tiled with only hexagons as well as TCNTs with pentagons and heptagons that are known to sustain strong ring currents. ${ }^{53}$ In Sections 2.1 and 2.2, we discuss the construction and optimization of the molecular structures, respectively. The methods used to calculate magnetically induced current densities are presented in Section 2.3. The results are reported in Section 3, and the main conclusions are drawn in Section 4.

\section{COMPUTATIONAL METHODS}

2.1. Polyhex Torus Construction. The surface of a torus can be obtained from a planar parallelogram-shaped surface by joining two opposite edges without twisting to form an openended tube. The two ends of the tube are then connected to yield a torus. Tiling the surface of a torus with hexagons can be realized by cutting a parallelogram out of an infinite plane tiled with regular hexagons. The corners of the parallelogram lie at centers of hexagons, and the mapping onto the surface of the torus is done in such a way that all four corners are mapped onto the same point. ${ }^{54-56}$ We here limit the graph-theoretical discussion to the construction of TCNTs that consist of only hexagons, which are called polyhexes. When constructing TCNTs with an equal number of pentagons and heptagons, their positions have to be carefully chosen to obtain regular structures as those studied in this work.

The dual of a hexagonal tiling of a surface is a six-connected triangulation. As proved by Negami, ${ }^{57}$ every six-connected triangulation of a torus has a unique embedding. A parallelogram that is tiled with regular hexagons can be uniquely defined by its edge vectors $(m, n)$ and $(p, q)$ in the hexagonal lattice (Figure 1). ${ }^{54}$ The number of vertices is

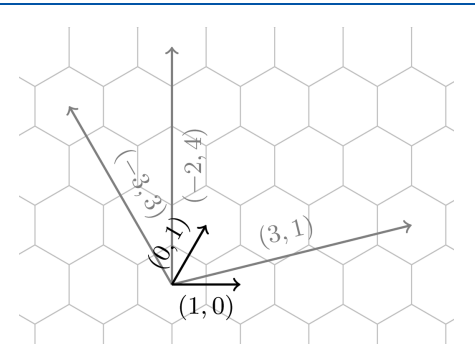

Figure 1. Two basis vectors as used in this paper as well as three examples for vectors $(m, n)$ or $(p, q)$. Note that different definitions of the basis vectors are used in the literature.

$2((m+n) q-n(p+q)) .{ }^{58}$ While $m, n, p$, and $q$ uniquely define the tiling of tori, the reverse is not true since each tiling can be generated by an infinite number of parameter sets. Exploiting this degeneracy renders parametrization of the tiling of a torus with only three integers feasible, which is less intuitive. ${ }^{57,58}$

The choice of $m, n, p$, and $q$ defines the minor radius $r$ and the major radius $R$ of the torus, where the minor radius is the radius of the tube that is bent to form a ring, whereas the length of the tube determines the major radius. The minor radius is equal to the length of the chiral lattice vector $(m, n)$ divided by $2 \pi$ or $2 \pi r=m^{2}+m n+n^{2}$. The major radius of the torus is equal to the length of the $(p, q)$ lattice vector times the sine of the angle between the $(m, n)$ and $(p, q)$ vectors divided by $2 \pi$.

The requirement to map the parallelogram onto the surface of the torus in such a way that all corners coincide implies that a general parallelogram has to be sheared to form a rectangle. Deviations from $\pi / 2$ of the angle between the two lattice vectors $(m, n)$ and $(p, q)$ lead to distortions of each hexagon when forming the torus. The angle between $(m, n)$ and $(p, q)$ can only be exactly $\pi / 2$ when the number of vertices is $4 n$ with integer $n$. The slightest deviation from $\pi / 2$ allows the construction of tori with vertex counts of $4 n+2$, whose hexagons are deformed.

A $2 \pi r \times 2 \pi R$ rectangle can be mapped onto the surface of a torus as

$$
\begin{aligned}
& x=\left(R+r_{x y} \cos (v)\right) \cos (w) \\
& y=\left(R+r_{x y} \cos (v)\right) \sin (w) \\
& z=r_{z} \sin (v)
\end{aligned}
$$

where $v$ and $w$ are angles corresponding to the radii $R$ and $r$, respectively.

The tube of the obtained torus has a circular cross section when $r=r_{x y}=r_{z}$. TCNTs with a large minor radii $r$ have been found to have ellipsoidal cross sections, ${ }^{8}$ which can be easily understood because such a deformation leads to more uniform bond lengths. Tori with ellipsoidal cross sections can be generated by reducing the minor radius $r_{x y}$ and increasing $r_{z}$ in eq 2. One of the main problems with the molecular structure of TCNTs is that $\mathrm{C}-\mathrm{C}$ bonds on the inside are very compressed, whereas those on the outside are often much longer than typical C-C single bonds. Small polyhex TCNTs are therefore thermodynamically unstable. ${ }^{8}$ More equal bond lengths are obtained by introducing heptagons on the inside and pentagons on the outside of the torus. The code we have used for generating arbitrary polyhex TCNTs is freely available. ${ }^{59}$

2.2. Structure Optimization. A practical method for obtaining good starting structures for large TCNTs is optimizing the structure in a reduced space with a few degrees of freedom. The largest polyhex TCNTs studied in this work were preoptimized in a two-parameter space by using the downhill simplex algorithm. One parameter is the isotropic scaling factor for the whole molecule, and the second parameter defines the extent of the elliptical distortion of the cross section of the TCNT. The vertical component $r_{z}$ and the horizontal component $r_{x y}$ of the minor radius are expressed as $r_{z}=\alpha r$ and $r_{x y}=2 r-\alpha r$, respectively, where $\alpha$ is the parameter that determines the shape of the cross section. The tube has a circular cross section when $\alpha=1$. Single-point energies were obtained with Turbomole ${ }^{60,61}$ by performing calculations at the density functional theory (DFT) level using the BeckePerdew (BP86) functional and split-valence quality basis sets augmented with polarization functions (def2-SVP). ${ }^{62-67}$ The accuracy of the molecular structures obtained in the twoparameter optimization was assessed by comparing the preoptimized structures and their energies with the corresponding results for the fully optimized ones. The average energy difference per carbon atom is about $1 \mathrm{mE}_{\mathrm{h}}$, showing 
Table 1. Number of Carbon Atoms $\left(N_{\mathrm{C}}\right)$, Lattice Vectors $(m, n),(p, q)$, Ideal Point Group and the Point Group Used as Symmetry Constraint in the Structure Optimization, HOMO-LUMO Gap, and Net Currents of the 13 Studied Polyhex TCNTs $^{a}$

\begin{tabular}{|c|c|c|c|c|c|c|}
\hline \multirow[b]{2}{*}{ series } & \multirow[b]{2}{*}{$N_{\mathrm{C}}$} & \multirow[b]{2}{*}{$(m, n),(p, q)$ parameters } & \multicolumn{2}{|c|}{ point group } & \multirow[b]{2}{*}{ HOMO-LUMO gap (eV) } & \multirow[b]{2}{*}{ ring current $(\mathrm{nA} / \mathrm{T})$} \\
\hline & & & ideal & optimized & & \\
\hline \multirow[t]{6}{*}{ (I) } & 480 & $(4,4),(-30,30)$ & $D_{30 \mathrm{~h}}$ & $C_{30 \mathrm{v}}$ & 0.20 & 84.4 \\
\hline & 576 & $(4,4),(-36,36)$ & $D_{36 \mathrm{~h}}$ & $C_{36 \mathrm{v}}$ & 0.18 & 112.8 \\
\hline & 672 & $(4,4),(-42,42)$ & $D_{42 \mathrm{~h}}$ & $C_{42 \mathrm{v}}$ & 0.14 & 143.4 \\
\hline & 768 & $(4,4),(-48,48)$ & $D_{48 \mathrm{~h}}$ & $C_{48 \mathrm{v}}$ & 0.13 & 171.3 \\
\hline & 864 & $(4,4),(-54,54)$ & $D_{54 \mathrm{~h}}$ & $C_{54 v}$ & 0.11 & 200.7 \\
\hline & 960 & $(4,4),(-60,60)$ & $D_{60 \mathrm{~h}}$ & $C_{60 \mathrm{v}}$ & 0.10 & 235.7 \\
\hline \multirow[t]{3}{*}{ (II) } & 588 & $(7,0),(-21,42)$ & $D_{21 \mathrm{~h}}$ & $D_{21 \mathrm{~h}}$ & 0.16 & 9.5 \\
\hline & 756 & $(7,0),(-27,54)$ & $D_{27 \mathrm{~h}}$ & $D_{27 \mathrm{~h}}$ & 0.31 & 1.7 \\
\hline & 924 & $(7,0),(-33,66)$ & $D_{33 \mathrm{~h}}$ & $D_{33 \mathrm{~h}}$ & 0.17 & 1.1 \\
\hline \multirow[t]{4}{*}{ (III) } & 756 & $(6,3),(-36,45)$ & $D_{9}$ & $D_{9}$ & 0.25 & -1.1 \\
\hline & 2016 & $(6,0),(-84,168)$ & $D_{84 \mathrm{~h}}$ & $D_{42 \mathrm{~h}}$ & & \\
\hline & 2016 & $(6,3),(-96,120)$ & $D_{24}$ & $D_{8}$ & 0.07 & 292.3 \\
\hline & 2016 & $(6,6),(-84,84)$ & $D_{84 \mathrm{~h}}$ & $D_{42 \mathrm{~h}}$ & 0.11 & 330.5 \\
\hline
\end{tabular}

${ }^{a}$ The first series (I) consists of six $(n, n)$ armchair TCNTs that permit a Clar structure. The second series (II) consist of three $(n, 0)$ zigzag TCNTs. Series (III) consists of four TCNTs that have Clar structures. The first and third of series (III) are chiral, the second has a zigzag structure, and the fourth is an armchair TCNT.

Table 2. Number of Carbon Atoms $\left(N_{\mathrm{C}}\right)$, Structural Parameters, Point Group, HOMO-LUMO Gap, and Net Currents of 9 Chuang-Armchair (Series (IV)), 5 Chuang-Zigzag ${ }^{13}$ (Series (V)), and 10 Dunlap-Armchair-Zigzag ${ }^{3}$ (Series (VI) and (VII)) TCNTs

\begin{tabular}{|c|c|c|c|c|c|}
\hline series & $N_{\mathrm{C}}$ & structural parameters & point group & HOMO-LUMO gap (eV) & ring current $(\mathrm{nA} / \mathrm{T})$ \\
\hline \multirow[t]{9}{*}{ (IV) } & 192 & $(2,1,1,1),(1,0)$ & $D_{6 \mathrm{~h}}$ & 1.33 & -3.5 \\
\hline & 288 & $(2,1,1,2),(1,0)$ & $D_{6 \mathrm{~h}}$ & 0.32 & -0.6 \\
\hline & 384 & $(2,1,1,3),(1,0)$ & $D_{6 \mathrm{~h}}$ & 0.29 & -2.4 \\
\hline & 480 & $(2,1,1,4),(1,0)$ & $D_{6 \mathrm{~h}}$ & 0.52 & -2.5 \\
\hline & 576 & $(2,1,1,5),(1,0)$ & $D_{6 \mathrm{~h}}$ & 0.04 & 57.7 \\
\hline & 672 & $(2,1,1,6),(1,0)$ & $D_{6 \mathrm{~h}}$ & 0.40 & -2.2 \\
\hline & 768 & $(2,1,1,7),(1,0)$ & $D_{6 \mathrm{~h}}$ & 0.23 & -3.8 \\
\hline & 864 & $(2,1,1,8),(1,0)$ & $D_{6 \mathrm{~h}}$ & 0.09 & -1.9 \\
\hline & 960 & $(2,1,1,9),(1,0)$ & $D_{6 \mathrm{~h}}$ & 0.31 & -2.7 \\
\hline \multirow[t]{5}{*}{ (V) } & 252 & $(1,2,2,1),(1,1)$ & $D_{6 \mathrm{~h}}$ & 0.26 & -8.4 \\
\hline & 420 & $(1,2,2,2),(1,1)$ & $D_{6 \mathrm{~h}}$ & 0.07 & -94.0 \\
\hline & 588 & $(1,2,2,3),(1,1)$ & $D_{6 \mathrm{~h}}$ & 0.25 & -12.9 \\
\hline & 756 & $(1,2,2,4),(1,1)$ & $D_{6 \mathrm{~h}}$ & 0.36 & -3.5 \\
\hline & 924 & $(1,2,2,5),(1,1)$ & $D_{6 \mathrm{~h}}$ & 0.33 & -1.5 \\
\hline \multirow[t]{6}{*}{ (VI) } & 480 & $2(4,4), 1(8,0)$ & $D_{6 \mathrm{~h}}$ & 0.16 & 73.7 \\
\hline & 576 & $3(4,4), 1(8,0)$ & $D_{6 \mathrm{~h}}$ & 0.18 & 117.8 \\
\hline & 672 & $4(4,4), 1(8,0)$ & $D_{6 \mathrm{~h}}$ & 0.08 & -94.6 \\
\hline & 768 & $5(4,4), 1(8,0)$ & $D_{6 \mathrm{~h}}$ & 0.19 & 149.0 \\
\hline & 864 & $6(4,4), 1(8,0)$ & $D_{6 \mathrm{~h}}$ & 0.08 & 172.3 \\
\hline & 960 & $7(4,4), 1(8,0)$ & $D_{6 \mathrm{~h}}$ & 0.10 & 177.6 \\
\hline \multirow[t]{4}{*}{ (VII) } & 672 & $2(4,4), 2(8,0)$ & $D_{6 \mathrm{~h}}$ & 0.09 & 94.6 \\
\hline & 768 & $3(4,4), 2(8,0)$ & $D_{6 \mathrm{~h}}$ & 0.12 & 119.2 \\
\hline & 864 & $2(4,4), 3(8,0)$ & $D_{6 \mathrm{~h}}$ & 0.03 & 101.1 \\
\hline & 960 & $3(4,4), 3(8,0)$ & $D_{6 \mathrm{~h}}$ & 0.07 & -4.1 \\
\hline
\end{tabular}

that rather accurate molecular structures can be obtained by optimizing the two structural parameters.

The meta-generalized-gradient approximation exchangecorrelation functional (TPSS), ${ }^{68}$ def2-SVP basis sets, and the $\mathrm{m} 3$ integration grid were used in the DFT optimization of the molecular structures of the TCNTs of series (I)-(II) and (IV)-(VII). ${ }^{65-69}$ The molecular structures of the TCNTs of series (III) were optimized at the BP86/def2-SVP level using the $\mathrm{m} 3$ integration grid. ${ }^{62-67,69}$ The semiempirical D3-BJ term was used for considering dispersion interactions. ${ }^{70}$ The geometry optimizations were constrained by using point group symmetries. In most cases, the ideal point group was chosen, that is, the highest point group permitted by the bond graph. As specified in Tables 1 and 2, a lower point group was chosen in some cases for technical reasons. The Cartesian coordinates of the optimized structures are given in the Supporting Information.

2.3. Magnetically Induced Current Density. Molecules exposed to an external magnetic field sustain magnetically induced current densities, which are the fingerprint of 
molecular magnetic properties that can be obtained by integrating the corresponding Biot-Savart expression. ${ }^{71,72}$ The magnetically induced current density susceptibility, which we, in the following, call current density, were calculated in the limit of zero magnetic field strength using the gaugeincluding magnetically induced current (GIMIC) method. $^{72-75}$ GIMIC uses basis set information, the atomic orbital density matrix, and the magnetically perturbed atomic orbital density matrices as input data, which are obtained by performing nuclear magnetic resonance (NMR) shielding calculations. The GIMIC program is an open-source tool for calculating and analyzing current densities in open- and closedshell molecules. Details about the use of the GIMIC method and its applications can be found on $\mathrm{GitHub}^{76}$ and in the reviews by Sundholm et al. ${ }^{72,75,77}$

GIMIC is interfaced to a number of electronic structure codes such as Turbomole or Gaussian. ${ }^{76,78}$ The NMR shieldings and current densities were calculated ${ }^{60,61}$ at the DFT level using gauge-including atomic orbitals (GIAO) to ensure gauge origin independence and a rapid basis-set convergence. $^{79,80}$ The TPSS/def2-SVP level was used in the NMR shielding and current-density calculations. ${ }^{66,68,81}$ The performance of different functionals was also investigated by performing current-density calculations using the M06-2X,

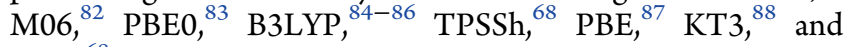
TPSS $^{68}$ functionals.

When a magnetic field is directed perpendicular to a molecular ring, the current density can flow around the ring, forming a ring current whose strength can be used for assessing the degree of aromaticity or used as a general measure of electron delocalization. The net ring current can flow in the diatropic, that is, classical direction, or in the paratropic (opposite) direction. Molecules sustaining net diatropic ring currents are aromatic, whereas antiaromatic molecular rings are dominated by paratropic ring currents. Nonaromatic molecules can sustain diatropic and paratropic ring currents of equal magnitude.

In toroidal structures, the induced current density can be decomposed into one vector component along the main ring of the torus and a second one that flows around the cross section of the nanotube. Helical current densities appear when there are nonzero net current strengths in both directions.

The strength of the ring current can be determined by performing numerical integration of the current density passing chosen integration planes through the molecule or through planes that cut chemical bonds. Here, the strength of the ring current of the major ring of the TCNTs has been determined by placing the integration plane starting from the center of the torus and extending at least $8 \mathrm{bohr}$ from the outer edge of the molecule. Detailed investigations of the ring current passing individual chemical bonds are not relevant because, for molecular cages and rings, the ring current does not necessarily follow chemical bonds; instead, the current pathway is determined by the topology of the molecule. ${ }^{47,89-98}$

When the surface of a torus lies in the $x y$ plane, the net ring current around the cross section of the nanotube can be obtained by integrating the current density using a cylindrical grid that covers only the positive part of the $z$ coordinate and intersects the torus. For TCNTs with rotational symmetry, it is sufficient to integrate over only a fraction of the grid. For sufficiently high rotational symmetry, the remaining arc can be replaced by a straight line, implying that a planar integration grid can, in practice, be employed.
The current density and its pathways can be qualitatively analyzed by visualizing the current density on a threedimensional (3D) grid using visualization programs such as Paraview. ${ }^{99}$ Paraview can also provide contour plots by visualizing the current density passing a given plane. Line integral convolution (LIC) plots can be used for tracing current vortices with a color scheme representing the current strength. The figures were prepared using Gnuplot, VMD, GIMP, and Paraview. ${ }^{99-102}$

\section{RESULTS AND DISCUSSION}

3.1. Investigated Structures. 3.1.1. Polyhex TCNTs. We have studied four polyhex-zigzag $(n, 0)$ TCNT structures, seven polyhex-armchair $(n, n)$, and two polyhex-chiral $(6,3)$ structures. The polyhex-armchair TCNTs have acene-like structures along the main ring of the torus. Ten of the studied TCNTs have Clar structures. Clar structures exist in TCNTs when $(m-n) \bmod 3=0 \wedge(p-q) \bmod 3=0 .{ }^{54}$ The studied polyhex TCNTs have $4 n \pi$ electrons, and the hexagons are not distorted. The role of Hückel's aromaticity rule was assessed by performing current density calculations on TCNTs with $4 n$ and $[4 n+2] \pi$ electrons. The calculations on TCNT with 500 , 748, and $1000(4 n)$ carbon atoms and 502, 750, and 1002 (4n +2 ) carbon atoms showed that they sustain practically the same ring-current strength. Therefore, we did not study TCNTs with distorted hexagons more extensively.

While a straight carbon nanotube $(\mathrm{CNT})$ with any $(m, n)$ can be realized with equal and ideal $\mathrm{C}-\mathrm{C}$ bond lengths, this is not possible for TCNTs that consist of hexagons only because the inner and outer perimeters have significantly different lengths. Therefore, large $r$ and small $R$ lead to the largest deviation from ideal bond lengths, while in the limit of thin tubes or for large $R$, little strain remains. Some of the smallest studied polyhex tori are highly strained. The shortest $\mathrm{C}-\mathrm{C}$ bonds inside the tori are much shorter than the optimal $\mathrm{C}-\mathrm{C}$ distance of about $142 \mathrm{pm}$, and the longest $\mathrm{C}-\mathrm{C}$ bonds on the outside of the tori are much longer. Since the smallest polyhex tori lie very high in energy as compared to $\mathrm{I}_{\mathrm{h}}-\mathrm{C}_{60}$ fullerene, the optimized structures will most likely break down when one does not impose any symmetry constraints.

The stress can, to some extent, be relieved by distorting the cross section of the tube toward an ellipse such that it is elongated perpendicular to the torus and compressed in the radial direction, which reduces the length difference between the inner and outer perimeters. This effect has been previously described and quantified based on molecular dynamics simulations. ${ }^{8}$ Here, we confirm it at the DFT level. The cross section of the tori is able to become elliptical in the geometry optimizations because the structures are constrained only with respect to the point group symmetry.

The stress can also be released by forming dents on the inside of a torus, leading to a longer inner perimeter, while the length of the outer perimeter remains unchanged. This stressreleasing mechanism has previously been described for CNTs $^{103}$ and TCNTs. ${ }^{8,104}$ The chiral $\mathrm{C}_{756}(m, n, p, q)=(6$, $3,-36,45)$ TCNT was found to have dents along the inner perimeter when only the point group symmetry constraint was imposed in the DFT optimization of the structure. The employed structural parameters and point groups as well as the obtained HOMO-LUMO gaps of the studied polyhex TCNTs are given in Table 1.

Three $\mathrm{C}_{2016}$ TCNTs with different $(m, n)$ parameters have been studied. The TCNT structure with $(m, n)=(6,0)$ does 
not have any energy gap between the highest occupied molecular orbital (HOMO) and the lowest unoccupied molecular orbital (LUMO) for the assumed point group, implying that nuclear shieldings and current densities cannot be calculated for it. The armchair $\mathrm{C}_{2016}$ TCNT with $(m, n)=$ $(6,6)$ has a slightly larger HOMO-LUMO gap of $0.11 \mathrm{eV}$ than that obtained for the intermediate chiral structure with $(m, n)=(6,3)$, whose HOMO-LUMO gap is $0.07 \mathrm{eV}$. The smaller $\mathrm{C}_{756}$ TCNT with $(m, n)=(6,3)$ has a HOMOLUMO gap of $0.25 \mathrm{eV}$. Polyhex CNTs with Clar structures have previously been found to lack a HOMO-LUMO gap, whereas the corresponding polycyclic aromatic hydrocarbons (PAH) have large HOMO-LUMO gaps. ${ }^{105}$ Isotropic distortions of CNT lead to increasing HOMO-LUMO gaps. ${ }^{106}$ The molecular structures of some of the studied polyhex TCNTs are shown in Figure 2.

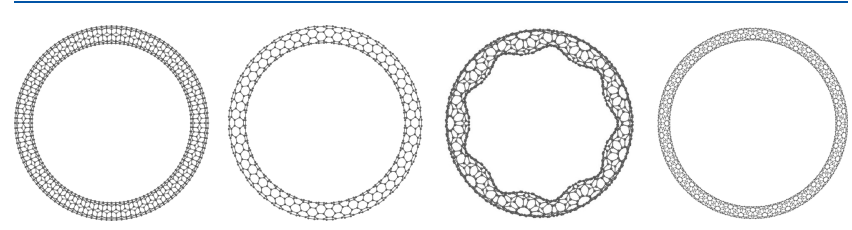

Figure 2. Structures of $\mathrm{C}_{960}$ of series (I), $\mathrm{C}_{924}$ of series (II), $\mathrm{C}_{756}$ of series (III), and $\mathrm{C}_{2016}(6,3)$ of series (III). The rest of the investigated polyhex TCNT structures is depicted in the Supporting Information.

3.1.2. TCNTs with Pentagons and Heptagons. More stable TCNTs with nearly equal $\mathrm{C}-\mathrm{C}$ distances can be obtained by introducing pentagons and heptagons. ${ }^{3-14,53}$ Here, we limit the discussion to two classes of TNCTs with pentagons and heptagons. They are labeled "Dunlap" and "Chuang" after the authors of refs 3 and 13, respectively, where the structures are described in detail. The Chuang TCNTs consist of six segments of straight nanotubes, and at each joint, there are two pentagons on the outside and two heptagons on the inside to reduce the strain. The point group is at least $D_{n}$. This class of TCNTs is characterized by six integers, $\left(n_{75}, n_{77}, n_{55}, s\right),(m$, $n$ ), where $n_{75}$ is the radial extent of one segment, $n_{77}$ is the inner height, $n_{55}$ is the outer height, $s$ is the length of each segment, and $(m, n)$ is the chiral vector. For explanatory diagrams, see ref 13 . The Dunlap TCNTs consist of 12 segments, which are alternatingly armchair and zigzag; that is, the point group is $D_{6 \mathrm{~h}}$. This class of TCNTs can be characterized by six integers, $s_{z}\left(m_{z}, n_{z}\right), s_{a}\left(m_{a}, n_{a}\right)$, which are the lengths and the chiral vectors of the zigzag-like segment and the armchair-like segment, respectively. There are similar constructions where the number of segments can be chosen to be any even number. ${ }^{4}$ However, we limit our study to TCNTs with 12 segments. The molecular structures of some of the studied Chuang and Dunlap TCNTs are shown in Figure 3. The employed structural parameters and point groups as well as the obtained HOMO-LUMO gaps and net currents of the studied Chuang and Dunlap TCNTs are given in Table 2.

The binding energy per carbon atom calculated for the polyhex series (I) and (II) in Figure 4 shows that the polyhex TCNTs are much higher in energy than the Chuang and Dunlap TCNTs with pentagons and heptagons. With increasing size of the TCNT, the energy difference between the two classes decreases. The TCNTs with pentagons and heptagons have almost the same binding energy per carbon atom, showing that most of the strain has been released.

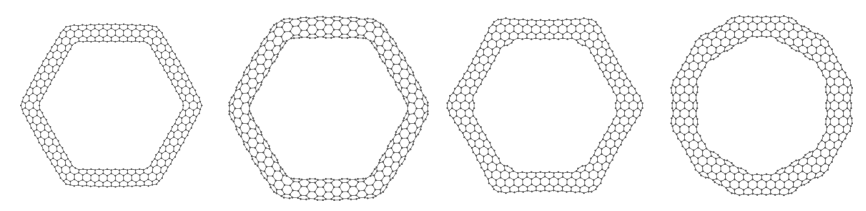

Figure 3. Structures of $\mathrm{C}_{960}$ of series (IV), $\mathrm{C}_{924}$ of series (V), $\mathrm{C}_{960}$ of series (VI), and $\mathrm{C}_{960}$ of series (VII). The rest of the investigated Chuang and Dunlap TCNT structures is depicted in the Supporting Information.

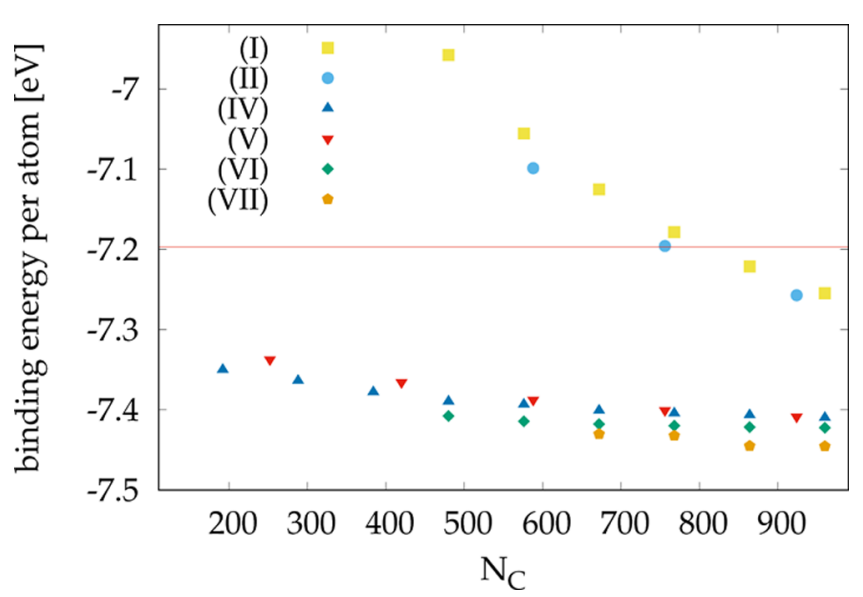

Figure 4. Binding energy per carbon atom for the studied TCNTs of series (I) -(II) and (IV)-(VII). The value for the icosahedral $\mathrm{C}_{60}$ fullerene calculated at the same level of theory (i.e., TPSS/def2-SVP) is used as a reference shown with the horizontal line.

Among the considered classes, the TCNTs of series (VII) are the most stable ones.

The calculations on TCNTs with pentagons and heptagons are summarized in Table 2. The calculated HOMO-LUMO gaps for them do not show any systematic trends. The HOMO-LUMO gaps for the Chuang-armchair structures varies from 0.04 to $1.33 \mathrm{eV}$, which is very similar to the ones obtained for the Chuang-zigzag TCNTs. For this class of TCNT, the armchair and zigzag TCNTs have very similar electronic properties. The HOMO-LUMO gaps of the studied Dunlap-armchair TCNTs are in the range of 0.03$0.19 \mathrm{eV}$. The HOMO-LUMO gap of the larger TCNTs is generally somewhat smaller than that of the smaller TCNTs.

The electronic properties of series (I) and (VI) TCNTs are similar because series (VI) consists of an increasing extent of armchair segments and may be considered as stabilized versions of the $(n, n)$ polyhexes. The band gap of infinite CNTs is zero when $(m-n) \bmod 3=0$. The band gap grows with the inverse of the diameter of the CNT. ${ }^{105,107,108}$ For TCNTs, one can expect a very small HOMO-LUMO gap when $(m-n) \bmod 3=0 \wedge(p-q) \bmod 3=0 .{ }^{54}$ The HOMO-LUMO gap of TCNTs will ideally grow with decreasing $r$ and $R$ as well as for TCNTs with elliptical cross sections. However, the long and short $\mathrm{C}-\mathrm{C}$ distances of the small TCNTs will also affect the HOMO-LUMO gap. Vanishing HOMO-LUMO gaps are expected for large polyhex-armchair TCNTs as for the corresponding CNTs. Calculations on the largest TCNTs of series (I) yield HOMO-LUMO gaps that approach zero. The three TCNTs of series (II) have a nonvanishing HOMO-LUMO gap. The TCNTs of series (III) are predicted to lack HOMOLUMO gaps because they are of Clar type. Deviations from the 
symmetry rule of the CNTs may be explained by the small $r$ and the elliptical cross section of the TCNTs. Especially, the smallest TCNT in series (III) has a very elliptic cross section with dents along the inner perimeter, as shown in Figure 2.

3.2. Ring-Current Strengths. Calculations of the net ringcurrent strength of the TCNTs show that the polyhex-armchair $(4,4),(p, q)$ TCNTs sustain very strong diatropic ring currents that increase with the size of the TCNT. The obtained ringcurrent strengths are summarized in Table 1 . The largest ringcurrent strength of series (I) was obtained for $\mathrm{C}_{960}$, which sustains a current strength of $235.7 \mathrm{nA} / \mathrm{T}$. The polyhex-zigzag $(7,0),(p, q)$ TCNTs in series (II) sustain weaker net ring currents whose strengths are only $1.1-9.5 \mathrm{nA} / \mathrm{T}$, even though the HOMO-LUMO gap is not much wider than for the polyhex-armchair TCNTs of series (I).

Most of the Chuang-armchair TCNTs in series (IV) and (V) sustain weak paratropic ring currents. The absolute value of the ring-current strength is generally smaller than $10 \mathrm{nA} / \mathrm{T}$. There are only three exceptions among the 14 studied Chuangarmchair TCNTs. $\mathrm{C}_{576}(2,1,1,5),(1,0)$ in series $($ IV) is the only one among them with a net diatropic ring current. The $\mathrm{C}_{420}(1,2,2,1),(1,1) \mathrm{TCNT}$ in series $(\mathbf{V})$ sustains a strong paratropic ring current of $-94.0 \mathrm{nA} / \mathrm{T}$. The paratropic ringcurrent strength of $\mathrm{C}_{588}(1,2,2,3),(1,1)$ in series $(\mathbf{V})$ is $-12.9 \mathrm{nA} / \mathrm{T}$. The obtained ring-current strengths can be compared with the reference current strength for benzene of $11.8 \mathrm{nA} / \mathrm{T} \cdot{ }^{31,75}$ The ring-current strength as a function of the size of the TCNTs is shown in Figure 5.

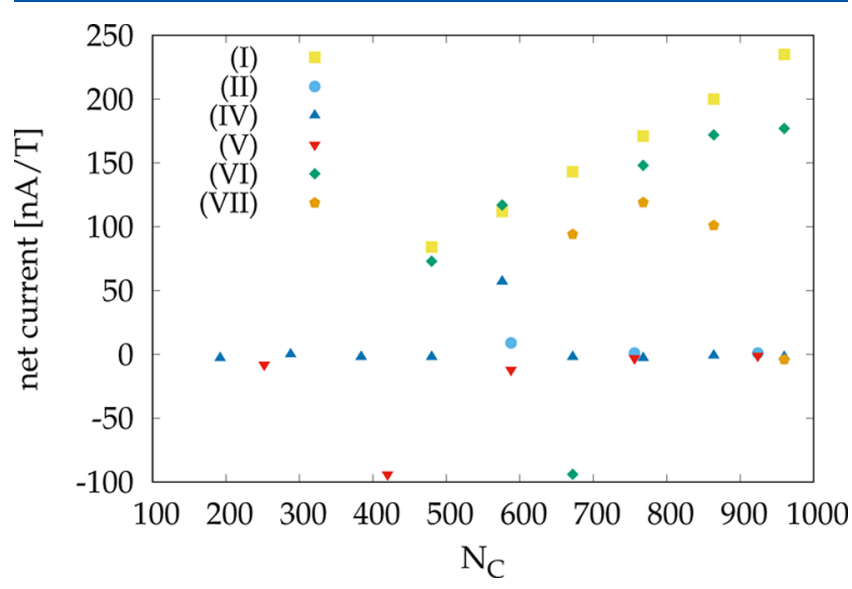

Figure 5. Strength of the ring current as a function of the size of the torus for the TCNTs of series (I) - (II) and (IV) $-($ VII).

The Dunlap-armchair-zigzag TCNTs with $s_{z}(4,4), 1(8,0)$ with $s_{z} \in\{2,3,4,5,6,7\}$ and $s_{z}(4,4), s_{a}(8,0)$ with $s_{z}, s_{a} \in\{2,3\}$ tend to sustain strong diatropic ring currents. Two exceptions are found among the 10 studied Dunlap-armchair-zigzag TCNTs. The $\mathrm{C}_{672} 4(4,4), 1(8,0)$ TCNT in series (VI) has a small HOMO-LUMO gap of $0.08 \mathrm{eV}$ and sustains a strong paratropic ring current of $-94.6 \mathrm{nA} / \mathrm{T}$. The $\mathrm{C}_{960} 3(4,4), 3(8$, 0) TCNT of series (VII) has a small HOMO-LUMO gap of $0.07 \mathrm{eV}$ and sustains a weak paratropic ring current of -4.1 $\mathrm{nA} / \mathrm{T}$. For the rest of the $s_{z}(4,4), 1(8,0)$ with $s_{z} \in$ $\{2,3,4,5,6,7\}$ TCNTs in series (VI), the ring-current strength increases with the size of the TCNT. The strongest ringcurrent strength of $177.6 \mathrm{nA} / \mathrm{T}$ was obtained for the $\mathrm{C}_{960}$ $7(4,4), 1(8,0)$ TCNT. Three of the studied TCNTs in the $s_{z}(4,4), s_{a}(8,0)$ with $s_{z}, s_{a} \in\{2,3\}$ series (VII) sustain diatropic ring currents of almost the same strength of about $100 \mathrm{nA} / \mathrm{T}$. The calculations on TCNTs with pentagons and heptagons are summarized in Table 2. The reason for the different behavior of the current density of the TCNTs in series (IV) and (V) as compared to the ones in series (VI) and (VII) is discussed in Section 3.3.

Haddon calculated the ring-current magnetic susceptibility of the $\mathrm{C}_{576} 3(4,4), 1(8,0)$ TCNT of series (VI) using finitefield London theory. ${ }^{53} \mathrm{He}$ obtained a value that is 130 times larger than the benzene value. The present calculations yield a net ring-current strength that is only 10 times larger than the ring-current strength of benzene, suggesting that the computational approach used by Haddon exaggerates the current strengths of TCNTs.

The calculations of the ring-current strengths of the TCNTs in series (III) show that the small chiral $\mathrm{C}_{756}$ TCNT sustains a very weak paratropic ring current of $-1.1 \mathrm{nA} / \mathrm{T}$, which can be explained by its highly elliptical cross section and dents along the inner perimeter. The ring-current strength of the large chiral $\mathrm{C}_{2016}$ TCNT is $292.3 \mathrm{nA} / \mathrm{T}$. The $\mathrm{C}_{2016}$ TCNT with an armchair structure sustains a diatropic ring current of 330.5 $\mathrm{nA} / \mathrm{T}$, whereas the corresponding zigzag structure has no HOMO-LUMO gap for the high-symmetry structure belonging to the $D_{42 \mathrm{~h}}$ point group. We did not reduce the symmetry constraints, even though optimization of its structure using less severe symmetry constraints might yield a HOMO-LUMO gap, because reducing the symmetry constraints significantly increases the computational costs and it can also lead to other problems.

The calculated current densities of the nonchiral TCNTs show that the current density mainly follows the carbon network along the major ring of the torus, which implies that the current density has hardly any vector component that forms a current pathway around the minor ring perpendicular to the major ring. However, the current density of the chiral $\mathrm{C}_{2016}$ TCNT has a very strong ring-current strength of 3307 $\mathrm{nA} / \mathrm{T}$ around the tube perpendicular to the torus, which is expected to result in a magnetically induced anapole moment. ${ }^{7,19,20,23,24}$

The calculated HOMO-LUMO gaps and ring-current strengths reported in Figure 6 show that TCNTs with a HOMO-LUMO gap smaller than $0.2 \mathrm{eV}$ sustain a strong net

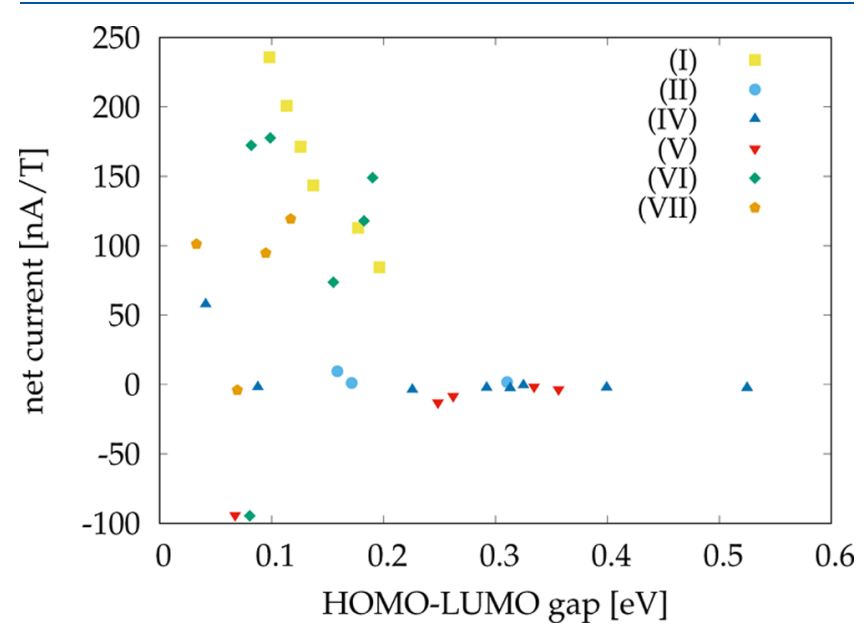

Figure 6. Net strength of the ring current as a function of the HOMO-LUMO gap for the TCNT of series (I)-(II) and (IV)(VII). 
ring current. However, this is a necessary condition but not a sufficient one. The polyhex TCNTs with armchair structures sustain stronger ring currents than the TCNTs with zigzag structures. The net current strength of TCNTs with an armchair structure increases with increasing radius of the torus. However, one must also bear in mind that acene-like structures might lead to open-shell singlet states that have multiconfiguration character and cannot be properly treated at the DFT level using today's functionals.

3.3. Current Density Flow. The current density flow in the TCNTs is discussed for some of the studied molecules. The current strength of the $3 \mathrm{D}$ vector field is depicted by stream lines that are colored based on the strength, where white means strong, and red represents weak current densities. The strength of the current density passing through a plane cutting the tube is shown in blue and red, where blue denotes the perpendicular vector component to the plane of diatropic ring currents, and red is the paratropic direction. Planes with excess of red indicate that the TCNT sustains a net paratropic ring current, and excess of blue indicates that it has a net diatropic ring current (cf. Figure 7), whereas equal patches of
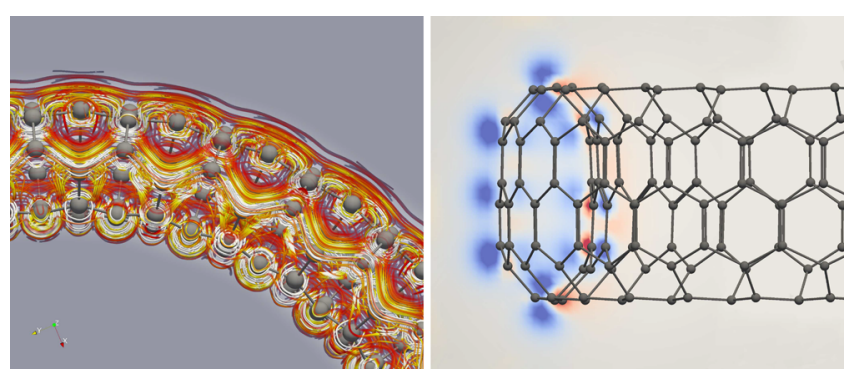

Figure 7. Current-density flow in $\mathrm{C}_{480}$ of series (I).

red and blue can be interpreted as local current-density flows leading to weak global ring-current strengths (cf. Figure 8). The pictures were made with Paraview. ${ }^{99}$
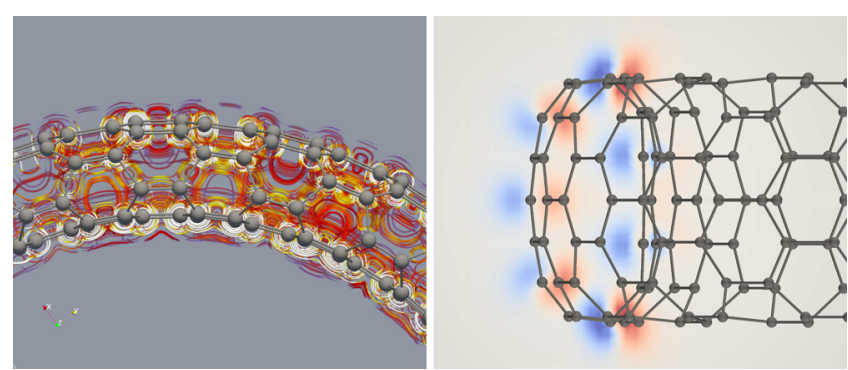

Figure 8. Current-density flow $\mathrm{C}_{588}$ of series (II).

In the TCNTs of series (I), there is a strong diatropic ring current on the outside, above, and below the torus (see Figure 7). The paratropic ring current on the inside is much weaker. There are prominent atomic currents around the atoms on the inside of the tube. The TCNTs of series (II) do not sustain any strong global ring currents. They have mainly local atomic and bond currents as seen in Figure 8 .

The $(6,3),(-96,120)$ and $(6,6),(-84,84)$ TCNTs sustain strong diatropic ring currents on the outside and inside of the torus, as seen in Figure 9. In contrast to the TCNTs of series (I) and (II), there is a much stronger current density flow inside the tube, which is probably due to the larger $r$.
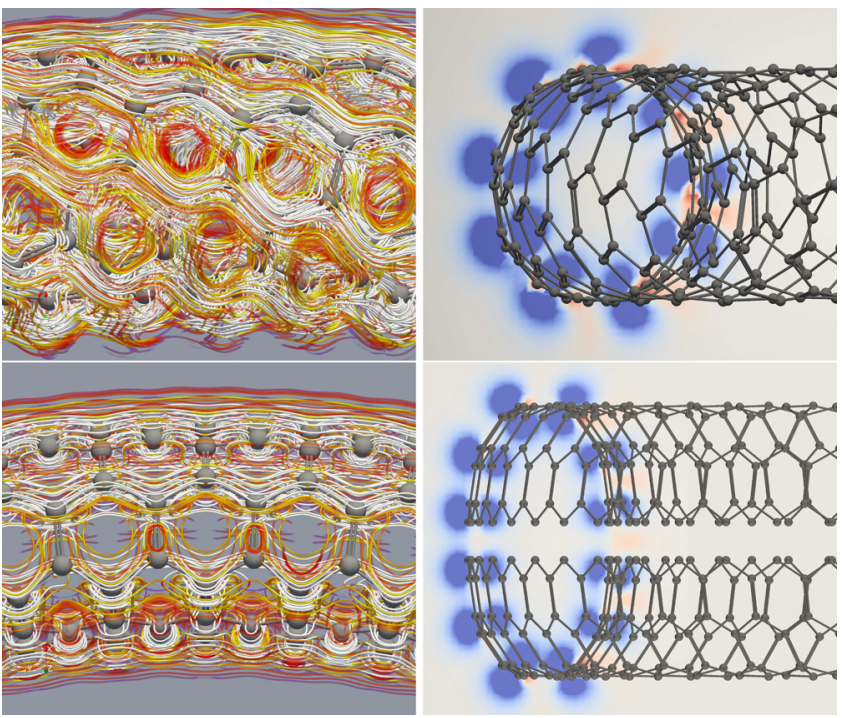

Figure 9. Current-density flow in $\mathrm{C}_{2016}(6,3),(-96,120)$ (top row) and $\mathrm{C}_{2016}(6,6),(-84,84)$ (bottom row) of series (III).

Most strikingly, the current density flow in the $(6,3),(-96$, 120) TCNT is helical; that is, it has a vector component of the current density parallel as well as perpendicular to the direction of the tube, as visualized in Figure 10.

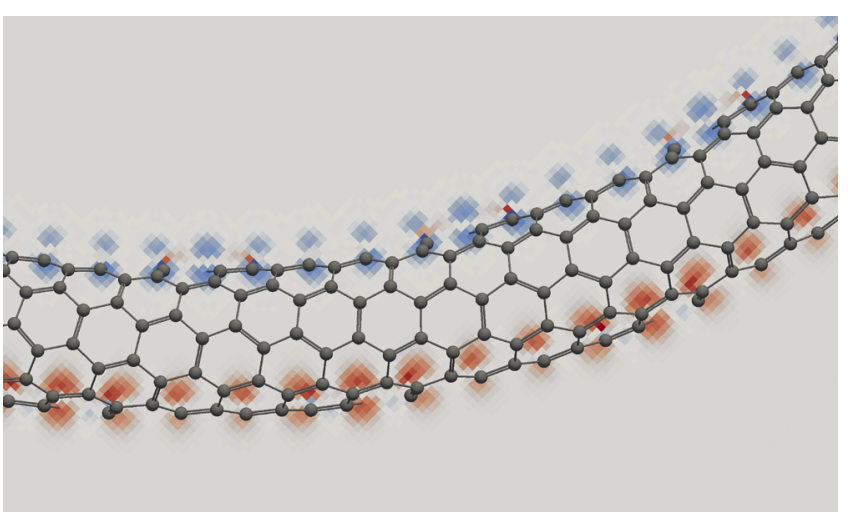

Figure 10. Horizontal slice through the chiral $C_{2016}(6,3),(-96$, $120)$. The plane is colored by the $z$-component of the current density, that is, the component perpendicular to the direction of the tube.

The current-density flows of $\mathrm{C}_{480}, \mathrm{C}_{576}$, and $\mathrm{C}_{672}$ of series (IV); $\mathrm{C}_{252}, \mathrm{C}_{420}, \mathrm{C}_{588}$, and $\mathrm{C}_{756}$ of series $(\mathrm{V})$; and $\mathrm{C}_{480}, \mathrm{C}_{576}$, $\mathrm{C}_{672}, \mathrm{C}_{768}$, and $\mathrm{C}_{864}$ of series (VI) have also been inspected; and two examples from each series are visualized. The current densities of the TCNTs of series (VII) are not visualized and discussed because they are similar to the ones for the TCNTs of series (VI).

The current density of the TCNTs of series (IV) in Figure 11 shows that there is a weak diatropic ring current on the outside and a weak paratropic current on the inside of the torus, which almost cancel each other, implying that net global ring current is weak. The only exception is $\mathrm{C}_{576}$, whose diatropic ring current is strong on the outside and reaches inward, leading to a net diatropic ring current of $57.7 \mathrm{nA} / \mathrm{T}$.

The members of series (V) have analogously two weak cancelling diatropic and paratropic ring currents, as shown in Figure 12. An exception is $\mathrm{C}_{420}$, whose paratropic current is much stronger on the inside and reaches further outward as 

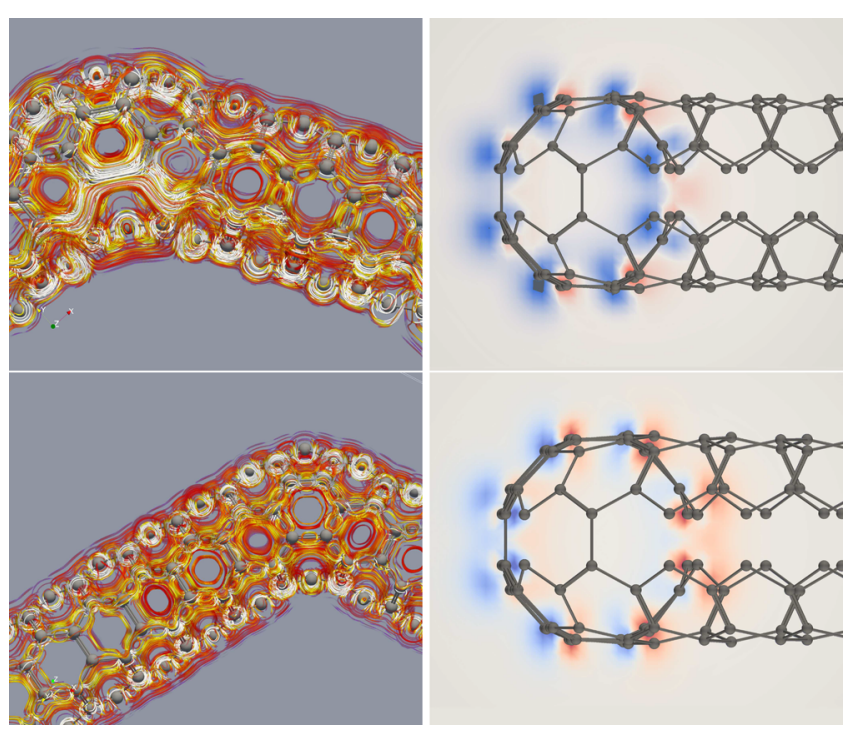

Figure 11. Current-density flow in $\mathrm{C}_{576}$ of series (IV) (top row) and $\mathrm{C}_{672}$ of series (IV) (bottom row).
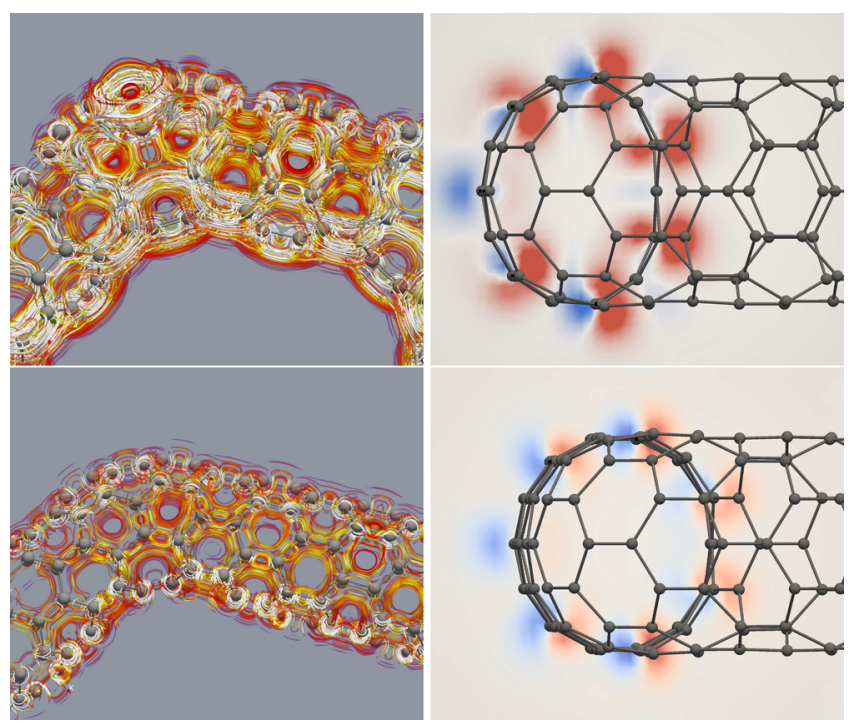

Figure 12. Current-density flow in $\mathrm{C}_{420}$ (top row) and $\mathrm{C}_{756}$ (bottom row) of series (V).

compared to the other TCNTs of series (V). The flow and strength of the diatropic ring current look like the ones for the other TCNTs of this series, leading to a strong net paratropic ring current of $-94.0 \mathrm{nA} / \mathrm{T}$.

For most of the TCNTs of series (VI), the diatropic ring current reaches very far inside the tube. The strongest pathway is on the top and bottom of the tube, as shown in Figure 13. The current density is weak at the hexagons on the outer edge of the tube because they are almost parallel to the direction of the applied magnetic field. This orientation is comparable to the case when the magnetic field is parallel to the ring of a benzene molecule. There is a very weak paratropic ring current on the inside of the torus. $\mathrm{C}_{672}$ is an exception, whose paratropic ring current is strong and reaches outward, leading to a net paratropic ring current of $-94.6 \mathrm{nA} / \mathrm{T}$. The strongest paratropic pathway follows the same route as in the other molecules of series (VI), but the direction is reversed.
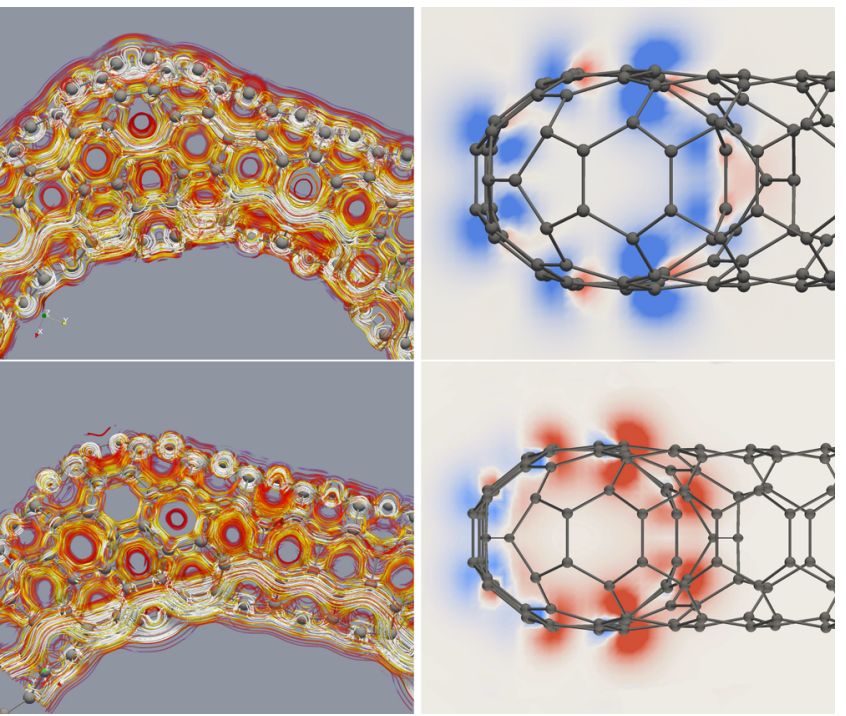

Figure 13. Current-density flow in $\mathrm{C}_{576}$ (top row) and $\mathrm{C}_{672}$ (bottom row) of series (VI).

Current-density plots reveal significant differences in the current density flow of the TCNTs in series (IV) and (VI). The locations of the pentagons and heptagons at the corners of the TCNT in series (IV) block the global current-density flow around the torus, leading to local ring currents along the edges between the corners. In $\mathrm{C}_{480}$ of series (VI), the heptagons along the inside edge of the torus do not prevent the main ring-current flow, allowing for a strong global diatropic ring current.

3.4. Functional Dependence. The strengths of the magnetically induced ring currents were calculated for the TCNTs of series (VI) using a number of popular density functionals. The obtained ring-current strengths as a function of the size of the TCNT are shown in Figure 14. The

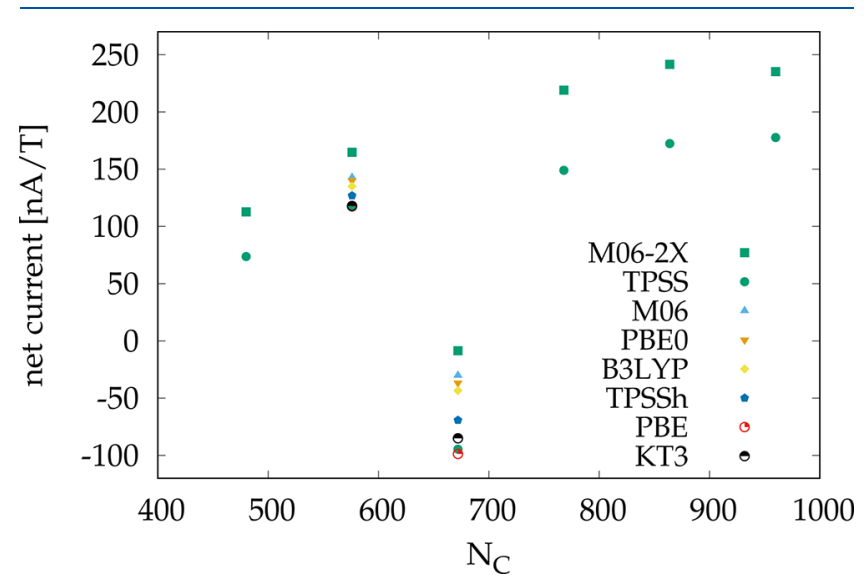

Figure 14. Strength of the ring current as a function of the employed DFT functional calculated for the TCNTs of series (VI).

employed functionals yield the same trend for the size dependence of the ring-current strength. The ring currents are diatropic and increase with the size of the TCNT, except for $\mathrm{C}_{672}$, which sustains a net paratropic ring current of -8.6 $\mathrm{nA} / \mathrm{T}$ at the M06-2X level, and a ring-current strength of $-94.6 \mathrm{nA} / \mathrm{T}$ is obtained with the TPSS functional. The strongest diatropic ring currents are obtained with the M06-2X functional that has the largest amount of HF exchange, 
whereas the least diatropic ring currents are obtained with the TPSS functional without $\mathrm{HF}$ exchange. The ring-current strengths of $\mathrm{C}_{576}$ and $\mathrm{C}_{672}$ of series (VI) as a function of the amount of HF exchange are shown in Figure 15.

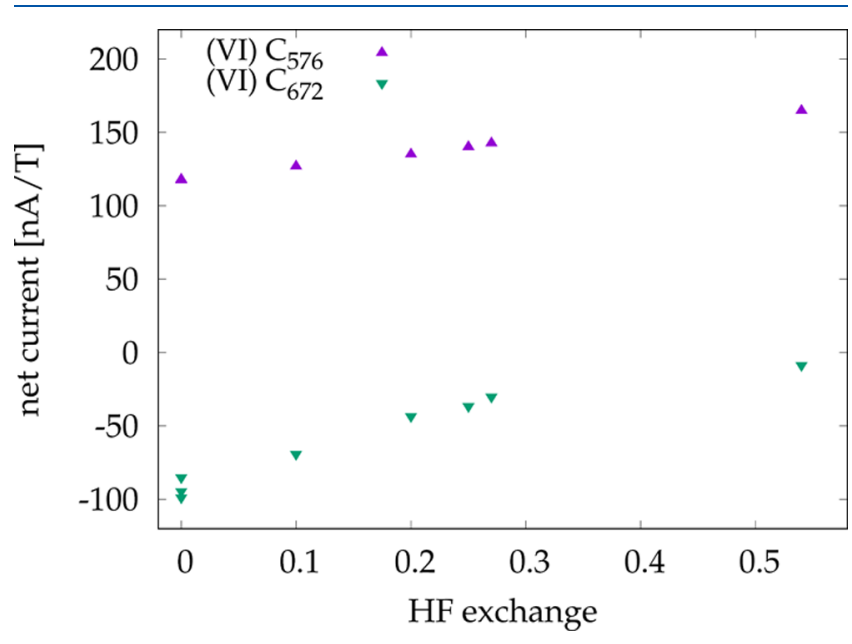

Figure 15. Ring-current strength calculated as a function of the amount of Hartree-Fock exchange in the DFT functional. The current strengths are reported for the $\mathrm{C}_{576}$ TCNT of series (VI) and the $\mathrm{C}_{672}$ TCNT of series (VI) that sustain strong diatropic and paratropic ring currents, respectively.

Previous studies have shown that ring-current strengths calculated for aromatic molecules ring currents using the B3LYP functional, which has $20 \% \mathrm{HF}$ exchange, are generally in good agreement with strengths obtained at the second-order Møller-Plesset perturbation theory level, ${ }^{52,109-112}$ whereas for very strongly antiaromatic molecules, B3LYP calculations overestimate the strength of the paratropic ring current by up to a factor of 3 . Based on a previous experience on currentdensity calculations, the current strengths of antiaromatic molecules should be calculated using a functional with a larger amount of HF exchange. However, the M06-2X functional seems to exaggerate the diatropic contribution to the ring currents. ${ }^{111}$ For antiaromatic molecules, the strengths of the paratropic ring currents calculated using the TPSS functional are probably in absolute value too large. However, the general trend for the size dependence of the ring-current strength of the aromatic molecules is obtained with the TPSS functional, which is used as the default functional in this study.

\section{SUMMARY AND CONCLUSIONS}

Molecular structures of large toroidal carbon nanotubes (TCNT) with polyhex structures have been constructed by using a method that has been described in detail in this work. The TCNT structures were first optimized using a twoparameter optimization method, where one parameter scales all the $\mathrm{C}-\mathrm{C}$ bonds of the constructed structures, and the second parameter allows an elliptical shape of the nanotube. The obtained structures are relatively accurate because the total energy of the molecular structures of the polyhex TCNTs optimized using the two-parameter method is very close to the energy of the structures that were fully optimized at the density functional theory (DFT) level. The structure optimizations show that the strain can be released by introducing dents on the inside of the torus and by making the cross section of the tube elliptical. TCNTs with pentagons and heptagons were also studied. Their initial structures were constructed following approaches described in the literature. ${ }^{3,13}$ The molecular structure of the studied TCNT was optimized at the DFT level using point groups with high symmetry, implying that they are not necessarily minima on the potential energy surface. Some of them are very high in energy because $\mathrm{C}-\mathrm{C}$ distances on the inside of the torus are much shorter than the optimal $\mathrm{C}-\mathrm{C}$ distance of, for example, $\mathrm{I}_{\mathrm{h}}-\mathrm{C}_{60}$ fullerene and the $\mathrm{C}-\mathrm{C}$ distances on the outside are very stretched.

Magnetically induced current densities have been calculated for the optimized molecular structures of the TCNTs using the gauge-including magnetically induced current (GIMIC) method. The current density flow has been quantified by determining the strength of the magnetically induced ring current around the torus. The ring-current strengths have been obtained by numerically integrating the current density passing a plane cutting the TCNT. The current densities have also been visualized by showing the main current density pathways in the TCNTs.

The calculations show that TCNTs with a HOMO-LUMO gap smaller than $0.2 \mathrm{eV}$ can sustain a strong diatropic ring current. This appears to be a necessary condition, whereas not all TCNTs with a small HOMO-LUMO gap sustain a strong net ring current in the presence of magnetic fields. The diatropic ring current of polyhex TCNTs with an armchair structure increases with increasing size of the torus, whereas the studied TCNTs with an zigzag structure sustain weak ring currents.

The 14 TCNTs with pentagons and heptagons that were constructed as suggested by Chuang et al. ${ }^{13}$ sustain paratropic ring currents with only one exception. The $\mathrm{C}_{576} \mathrm{TCNT}$ of series (IV) has a net diatropic ring current of $57.7 \mathrm{nA} / \mathrm{T}$. The strength of the paratropic ring current of these TCNTs is generally weak. However, the $\mathrm{C}_{420}$ TCNT of series (V) sustains a strong paratropic ring current of $-94.0 \mathrm{nA} / \mathrm{T}$.

The 10 TCNTs with pentagons and heptagons that were constructed as suggested by Dunlap ${ }^{3}$ sustain strong diatropic ring currents with two exceptions. The ring current of the $\mathrm{C}_{672}$ TCNT of series (VI) is strongly paratropic, and the $\mathrm{C}_{960}$ TCNT of series (VII) sustains a very weak paratropic ring current. In the series (VI) characterized by the $s_{z}(4,4), 1(8,0)$ with $s_{z} \in\{2,3,4,5,6,7\}$ parameters, the ring-current strength increases with the size of the torus. Regarding the TCNTs of series (VII), which is characterized by the $s_{z}(4,4), s_{a}(8,0)$ with $s_{z}, s_{a} \in\{2,3\}$ parameters, the ring-current strength is practically the same or about $100 \mathrm{nA} / \mathrm{T}$ with one exception. Visualization of the current density shows that the orientation of the pentagons and heptagons can block the global ring current, leading to strong localized ring currents. This is probably the main reason for the different ring-current strengths of the TCNTs in series (IV) and (V) as compared to the ones of the TCNTs in series (VI) and (VII).

In series (III), we investigated how the ring-current strength depends on the structure of the TCNT. Large polyhex TCNTs with 2016 carbon atoms were chosen to reduce the strain effects. The zigzag $\mathrm{C}_{2016}$ did not have any HOMO-LUMO gap for the assumed high-symmetry structure. The armchair $\mathrm{C}_{2016}$ TCNT sustains a ring current of $330.5 \mathrm{nA} / \mathrm{T}$, whereas a ring-current strength of $292.3 \mathrm{nA} / \mathrm{T}$ was obtained for the chiral $\mathrm{C}_{2016}(6,3),(-96,120)$ TCNT. The smaller chiral $\mathrm{C}_{756}(6,3)$, $(-36,45)$ TCNT has dents on the inside of the torus and does not sustain any significant ring current. 
The size of the TCNT must be large enough to sustain a ring current with a significant strength probably due to the large strain and abnormal $\mathrm{C}-\mathrm{C}$ distances for small ones. The $\mathrm{C}_{420}$ and $\mathrm{C}_{480}$ TCNTs are the smallest investigated molecules with a large ring-current strength. The calculations of the strength of the magnetically induced ring currents of the studied TCNT reveal some clear trends. However, several exceptions were obtained for the investigated TCNT series. The underlying reason for the discrepant behavior is not known.

The performance of eight DFT functionals was investigated by calculating the ring-current strength for the $\mathrm{C}_{576} 3(4,4)$, $1(8,0)$ TCNT that sustains a strong diatropic ring current and for $\mathrm{C}_{672} 3(4,4), 1(8,0)$, whose ring current is paratropic. The calculations show that the current density is systematically shifted toward stronger diatropicity when increasing the amount of Hartree-Fock exchange in the functional.

\section{ASSOCIATED CONTENT}

\section{S Supporting Information}

The Supporting Information is available free of charge on the ACS Publications website at DOI: 10.1021/acs.jpcc.9b03769.

Optimized Cartesian coordinates of all investigated molecules, pictures of all structures analoguous to Figures 2 and 3, and visualization of the integration planes used (PDF)

Molecular geometries of series (I)-(VII) (ZIP)

\section{AUTHOR INFORMATION}

\section{Corresponding Authors}

*E-mail: lukas.wirz@helsinki.fi (L.N.W.).

*E-mail: dage.sundholm@helsinki.fi (D.S.).

\section{ORCID}

Florian Weigend: 0000-0001-5060-1689

Lukas N. Wirz: 0000-0002-6577-7166

Maria Dimitrova: 0000-0002-0711-3484

Dage Sundholm: 0000-0002-2367-9277

\section{Author Contributions}

${ }^{\S}$ K.R. and L.N.W. contributed equally.

\section{Notes}

The authors declare no competing financial interest.

\section{ACKNOWLEDGMENTS}

L.N.W. thanks Alexander von Humboldt Foundation for a Feodor Lynen fellowship. K.R. acknowledges financial support from SFB 1176, project Q5. This work was supported by Magnus Ehrnrooth Foundation and by The Academy of Finland through projects 275845, 309394, and 314821. The authors acknowledge computational resources from CSC-the Finnish IT Center for Science. Computational resources from the Finnish Grid and Cloud Infrastructure (persistent identifier urn:nbn:fi:research-infras-2016072533) are also acknowledged. We thank Uwe Huniar for the advice concerning NMR shielding calculations.

\section{REFERENCES}

(1) Terrones, H.; Mackay, A. L. The geometry of hypothetical curved graphite structures. Carbon 1992, 30, 1251-1260.

(2) Schwerdtfeger, P.; Wirz, L. N.; Avery, J. The topology of fullerenes. Wiley Interdiscip. Rev.: Comput. Mol. Sci. 2015, 5, 96-145.
(3) Dunlap, B. I. Connecting carbon tubules. Phys. Rev. B 1992, 46, 1933-1936.

(4) Itoh, S.; Ihara, S. Toroidal forms of graphitic carbon. II. Elongated tori. Phys. Rev. B 1993, 48, 8323-8328.

(5) Ihara, S.; Itoh, S.; Kitakami, J.-i. Toroidal forms of graphitic carbon. Phys. Rev. B 1993, 47, 12908-12911.

(6) Meunier, V.; Lambin, P.; Lucas, A. A. Atomic and electronic structures of large and small carbon tori. Phys. Rev. B 1998, 57, 14886-14890.

(7) Ceulemans, A.; Chibotaru, L. F.; Fowler, P. W. Molecular Anapole Moments. Phys. Rev. Lett. 1998, 80, 1861-1864.

(8) Han, J. Energetics and structures of fullerene crop circles. Chem. Phys. Lett. 1998, 282, 187-191.

(9) Oh, D.-H.; Mee Park, J.; Kim, K. S. Structures and electronic properties of small carbon nanotube tori. Phys. Rev. B 2000, 62, $1600-1603$.

(10) Beuerle, F.; Herrmann, C.; Whalley, A. C.; Valente, C.; Gamburd, A.; Ratner, M. A.; Stoddart, J. F. Optical and Vibrational Properties of Toroidal Carbon Nanotubes. Chem. - Eur. J. 2011, 17, $3868-3875$.

(11) Diudea, M. V.; Kirby, E. C. The Energetic Stability of Tori and Single-Wall Tubes. Fullerene Sci. Technol. 2001, 9, 445-465.

(12) Cox, B. J.; Hill, J. M. New Carbon Molecules in the Form of Elbow-Connected Nanotori. J. Phys. Chem. C 2007, 111, 1085510860.

(13) Chuang, C.; Fan, Y.-C.; Jin, B.-Y. Generalized Classification Scheme of Toroidal and Helical Carbon Nanotubes. J. Chem. Inf. Model. 2009, 49, 361-368.

(14) Diudea, M. V.; Nagy, C. L. Periodic Nanostructures; Braun, T., Ed.; Developments in Fullerene Science; Springer: Dordrecht, 2007; Vol. 7.

(15) Sarkar, A.; Kroto, H. W.; Endo, M. HEMI-toroidal networks in pyrolytic carbon nanotubes. Carbon 1995, 33, 51-55.

(16) Endo, M.; Takeuchi, K.; Kobori, K.; Takahashi, K.; Kroto, H. W.; Sarkar, A. Pyrolytic carbon nanotubes from vapor-grown carbon fibers. Carbon 1995, 33, 873-881.

(17) Terrones, M.; Hsu, W. K.; Hare, J. P.; Kroto, H. W.; Terrones, H.; Walton, D. R. M.; Klinowski, J.; Mackay, A. L. Graphitic structures: from planar to spheres, toroids and helices. Philos. Trans. R. Soc. Lond. A 1996, 354, 2025-2054.

(18) Liu, J.; Dai, H.; Hafner, J. H.; Colbert, D. T.; Smalley, R. E.; Tans, S. J.; Dekker, C. Fullerene 'crop circles'. Nature 1997, 385, 780-781.

(19) Gray, C. G.; Karl, G.; Novikov, V. A. Magnetic multipolar contact fields: The anapole and related moments. Am. J. Phys. 2010, $78,936-948$

(20) Tellgren, E. I.; Fliegl, H. Non-perturbative treatment of molecules in linear magnetic fields: Calculation of anapole susceptibilities. J. Chem. Phys. 2013, 139, 164118.

(21) Berger, R. J. F. Prediction of a Cyclic Helical Oligoacetylene Showing Anapolar Ring Currents in the Magnetic Field. Z. Naturforsch. 2012, 67, 1127-1131.

(22) Ceulemans, A.; Fowler, P. W. Symmetry Extensions of Euler's Theorem for Polyhedral, Toroidal and Benzenoid Molecules. J. Chem. Soc., Faraday Trans. 1995, 91, 3089-3093.

(23) Pelloni, S.; Lazzeretti, P.; Monaco, G.; Zanasi, R. Magnetic-field induced electronic anapoles in small molecules. Rend. Lincei 2011, 22, $105-112$.

(24) Papasimakis, N.; Fedotov, V. A.; Marinov, K.; Zheludev, N. I. Gyrotropy of a Metamolecule: Wire on a Torus. Phys. Rev. Lett. 2009, 103, No. 093901.

(25) Hückel, E. Quantentheoretische Beiträge zum Benzolproblem I. Die Elektronenkonfiguration des Benzols und verwandter Verbindungen. Z. Phys. 1931, 70, 204-286.

(26) Hückel, E. Quantentheoretische Beiträge zum Benzolproblem II. Quantentheorie der induzierten Polaritäten. Z. Phys. 1931, 72, 310-337.

(27) Hückel, E. Grundzüge der Theorie ungesättigter und aromatischer Verbindungen; Verlag Chemie: Berlin, 1938. 
(28) von Eggers Doering, W.; Detert, F. L. Cycloheptatrienylium Oxide. J. Am. Chem. Soc. 1951, 73, 876-877.

(29) Pople, J. A. Molecular Orbital Theory of Aromatic Ring Currents. Mol. Phys. 1958, 1, 175-180.

(30) McWeeny, R. Ring Currents and Proton Magnetic Resonance in Aromatic Molecules. Mol. Phys. 1958, 1, 311-321.

(31) Fliegl, H.; Sundholm, D.; Taubert, S.; Jusélius, J.; Klopper, W. Magnetically Induced Current Densities in Aromatic, Antiaromatic, Homoaromatic, and Nonaromatic Hydrocarbons. J. Phys. Chem. A 2009, 113, 8668-8676.

(32) Gomes, J. A. N. F.; Mallion, R. B. Aromaticity and Ring Currents. Chem. Rev. 2001, 101, 1349-1384.

(33) Steiner, E.; Fowler, P. W. Ring currents in aromatic hydrocarbons. Int. J. Quantum Chem. 1996, 60, 609-616.

(34) Stępień, M.; Latos-Grażyński, L.; Sprutta, N.; Chwalisz, P.; Szterenberg, L. Expanded Porphyrin with a Split Personality: A Hückel-Möbius Aromaticity Switch. Angew. Chem., Int. Ed. 2007, 46, 7869-7873.

(35) Jux, N. The Porphyrin Twist: Hückel and Möbius Aromaticity. Angew. Chem., Int. Ed. 2008, 47, 2543-2546.

(36) Herges, R. Aromatics with a twist. Nature 2007, 450, 36-37.

(37) Rappaport, S. M.; Rzepa, H. S. Intrinsically Chiral Aromaticity.

Rules Incorporating Linking Number, Twist, and Writhe for HigherTwist Möbius Annulenes. J. Am. Chem. Soc. 2008, 130, 7613-7619.

(38) Randić, M. Aromaticity of polycyclic conjugated hydrocarbons. Chem. Rev. 2003, 103, 3449-3606.

(39) Taubert, S.; Sundholm, D.; Jusélius, J.; Klopper, W.; Fliegl, H. Calculation of magnetically induced currents in hydrocarbon nanorings. J. Phys. Chem. A 2008, 112, 13584-13592.

(40) Herges, R. Topology in Chemistry: Designing Möbius Molecules. Chem. Rev. 2006, 106, 4820-4842.

(41) Shin, J.-Y.; Kim, K. S.; Yoon, M.-C.; Lim, J. M.; Yoon, Z. S.; Osuka, A.; Kim, D. Aromaticity and photophysical properties of various topology-controlled expanded porphyrins. Chem. Soc. Rev. 2010, 39, 2751-2767.

(42) Solá, M. Why Aromaticity Is a Suspicious Concept? Why? Front. Chem. 2017, 5, 22.

(43) Hajgató, B.; Deleuze, M. S.; Ohno, K. Aromaticity of giant polycyclic aromatic hydrocarbons with hollow sites: Super ring currents in super-rings. Chem. - Eur. J. 2006, 12, 5757-5769.

(44) Tahara, K.; Yoshimura, T.; Sonoda, M.; Tobe, Y.; Williams, R. V. Theoretical studies on graphyne substructures: Geometry, aromaticity, and electronic properties of the multiply fused dehydrobenzo[12] annulenes. J. Org. Chem. 2007, 72, 1437-1442.

(45) Fliegl, H.; Sundholm, D. Aromatic Pathways of Porphins, Chlorins and Bacteriochlorins. J. Org. Chem. 2012, 77, 3408-3414.

(46) Kaipio, M.; Patzschke, M.; Fliegl, H.; Pichierri, F.; Sundholm, D. effect of fluorine substitution on the aromaticity of polycyclic hydrocarbons. J. Phys. Chem. A 2012, 116, 10257-10268.

(47) Peeks, M. D.; Claridge, T. D. W.; Anderson, H. L. Aromatic and antiaromatic ring currents in a molecular nanoring. Nature 2017, 541, 200-203.

(48) Cha, W.-Y.; Kim, T.; Ghosh, A.; Zhang, Z.; Ke, X.-S.; Ali, R.; Lynch, V. M.; Jung, J.; Kim, W.; Lee, S.; Fukuzumi, S.; Park, J. S.; Sessler, J. L.; Chandrashekar, T. K.; Kim, D. Bicyclic Baird-type aromaticity. Nature Chem. 2017, 9, 1243-1248.

(49) Valiev, R. R.; Fliegl, H.; Sundholm, D. Bicycloaromaticity and Baird-type bicycloaromaticity of dithienothiophene-bridged [34]octaphyrins. Phys. Chem. Chem. Phys. 2018, 20, 17705-17713.

(50) Dimitrova, M.; Fliegl, H.; Sundholm, D. The influence of heteroatoms on the aromatic character and the current pathways of $\mathrm{B}_{2} \mathrm{~N}_{2}$-dibenzo[a,e]pentalenes. Phys. Chem. Chem. Phys. 2017, 19, 20213-20223.

(51) Dimitrova, M.; Sundholm, D. The aromatic character of [10]annulenes and dicupra[10]annulenes from current density calculations. Phys. Chem. Chem. Phys. 2018, 20, 1337-1346.

(52) Wirz, L. N.; Dimitrova, M.; Fliegl, H.; Sundholm, D. Magnetically Induced Ring-Current Strengths in Möbius Twisted Annulenes. J. Phys. Chem. Lett. 2018, 9, 1627-1632.
(53) Haddon, R. C. Electronic Properties of Carbon Toroids. Nature 1997, 388, 31-32.

(54) Ceulemans, A.; Chibotaru, L. F.; Bovin, S. A.; Fowler, P. W. The electronic structure of polyhex carbon tori. J. Chem. Phys. 2000, 112, 4271-4278.

(55) Bovin, S. A.; Chibotaru, L. F.; Ceulemans, A. The quantum structure of carbon tori. J. Mol. Catal. A 2001, 166, 47-52.

(56) Koorepazan-Moftakhar, F.; Ashrafi, A. R.; Ori, O.; Putz, M. V. Exotic Properties of Carbon Nanomatter; Putz, M. V., Ori, O., Eds.; Carbon Materials: Chemistry and Physics; Springer: Dordrecht, 2015, Vol, 8; Chapter 6.

(57) Negami, S. Uniqueness and faithfulness of embedding of toroidal graphs. Discrete Math. 1983, 44, 161-180.

(58) Kirby, E. C.; Mallion, R. B.; Pollak, P. Toroidal polyhexes. J. Chem. Soc., Faraday Trans. 1993, 89, 1945-1953.

(59) Wirz, L. N. Generation of molecular structures of nano tori, https://github.com/lnw/nano-tori.

(60) Ahlrichs, R.; Bär, M.; Häser, M.; Horn, H.; Kölmel, C. Electronic Structure Calculations on Workstation Computers: The Program System TURBOMOLE. Chem. Phys. Lett. 1989, 162, 165169.

(61) Furche, F.; Ahlrichs, R.; Hättig, C.; Klopper, W.; Sierka, M.; Weigend, F. Turbomole. Wiley Interdiscip. Rev.: Comput. Mol. Sci. 2013, 4, 91-100.

(62) Vosko, S. H.; Wilk, L.; Nusair, M. Accurate Spin-Dependent Electron Liquid Correlation Energies for Local spin density calculations: a Critical Analysis. Can. J. Phys. 1980, 58, 1200-1211.

(63) Perdew, J. P. Density-functional approximation for the correlation energy of the inhomogeneous electron gas. Phys. Rev. $B$ 1986, 33, 8822-8824.

(64) Becke, A. D. Density-functional exchange-energy approximation with correct asymptotic behavior. Phys. Rev. A 1988, 38, 30983100.

(65) Schäfer, A.; Horn, H.; Ahlrichs, R. Fully Optimized Contracted Gaussian-Basis Sets for Atoms Li to Kr. J. Chem. Phys. 1992, 97, $2571-2577$.

(66) Weigend, F.; Ahlrichs, R. Balanced basis sets of split valence, triple zeta valence and quadruple zeta valence quality for $\mathrm{H}$ to $\mathrm{Rn}$ : Design and assessment of accuracy. Phys. Chem. Chem. Phys. 2005, 7, $3297-3305$

(67) Weigend, F. Accurate Coulomb-fitting basis sets for $\mathrm{H}$ to $\mathrm{Rn}$. Phys. Chem. Chem. Phys. 2006, 8, 1057-1065.

(68) Tao, J.; Perdew, J. P.; Staroverov, V. N.; Scuseria, G. E. Climbing the Density Functional Ladder: Nonempirical MetaGeneralized Gradient Approximation Designed for Molecules and Solids. Phys. Rev. Lett. 2003, 91, 146401.

(69) Treutler, O.; Ahlrichs, R. Efficient molecular numerical integration schemes. J. Chem. Phys. 1995, 102, 346.

(70) Grimme, S.; Antony, J.; Ehrlich, S.; Krieg, H. A consistent and accurate ab initio parametrization of density functional dispersion correction (DFT-D) for the 94 elements H-Pu. J. Chem. Phys. 2010, $132,154104$.

(71) Lazzeretti, P. Ring currents. Prog. Nucl. Magn. Reson. Spectrosc. 2000, 36, 1-88.

(72) Sundholm, D.; Fliegl, H.; Berger, R. J. F. Calculations of magnetically induced current densities: theory and applications. WIREs Comput. Mol. Sci. 2016, 639-678.

(73) Jusélius, J.; Sundholm, D.; Gauss, J. Calculation of Current Densities using Gauge-Including Atomic Orbitals. J. Chem. Phys. 2004, 121, 3952-3963.

(74) Taubert, S.; Sundholm, D.; Jusélius, J. Calculation of spincurrent densities using gauge-including atomic orbitals. J. Chem. Phys. 2011, 134, No. 054123 .

(75) Fliegl, H.; Taubert, S.; Lehtonen, O.; Sundholm, D. The gauge including magnetically induced current method. Phys. Chem. Chem. Phys. 2011, 13, 20500-20518.

(76) GIMIC, version 2.0, a current density program. Can be freely downloaded from https://github.com/qmcurrents/gimic. 
(77) Fliegl, H.; Valiev, R.; Pichierri, F.; Sundholm, D. Theoretical studies as a tool for understanding the aromatic character of porphyrinoid compounds. Chem. Modell. 2018, 14, 1-42.

(78) Rauhalahti, M.; Taubert, S.; Sundholm, D.; Liégeois, V. Calculations of current densities for neutral and doubly charged persubstituted benzenes using effective core potentials. Phys. Chem. Chem. Phys. 2017, 19, 7124-7131.

(79) Ditchfield, R. Self-consistent perturbation theory of diamagnetism: I. A gauge-invariant LCAO method for N.M.R. chemical shifts. Mol. Phys. 1974, 27, 789-807.

(80) Wolinski, K.; Hinton, J. F.; Pulay, P. Efficient implementation of the gauge-independent atomic orbital method for NMR chemical shift calculations. J. Am. Chem. Soc. 1990, 112, 8251-8260.

(81) Reiter, K.; Mack, F.; Weigend, F. Calculation of Magnetic Shielding Constants with meta-GGA Functionals Employing the Multipole-Accelerated Resolution of the Identity: Implementation and Assessment of Accuracy and Efficiency. J. Chem. Theory Comput. 2018, 14, 191-197.

(82) Zhao, Y.; Truhlar, D. G. The M06 suite of density functionals for main group thermochemistry, thermochemical kinetics, noncovalent interactions, excited states, and transition elements: two new functionals and systematic testing of four M06-class functionals and 12 other functionals. Theor. Chem. Acc. 2008, 120, 215-241.

(83) Perdew, J. P.; Ernzerhof, M.; Burke, K. Rationale for mixing exact exchange with density functional approximations. J. Chem. Phys. 1996, 105, 9982-9985.

(84) Becke, A. D. Density-functional thermochemistry. III. The role of exact exchange. J. Chem. Phys. 1993, 98, 5648-5652.

(85) Lee, C.; Yang, W.; Parr, R. G. Development of the ColleSalvetti correlation-energy formula into a functional of the electron density. Phys. Rev. B 1988, 37, 785-789.

(86) Becke, A. D. A new mixing of Hartree-Fock and local densityfunctional theories. J. Chem. Phys. 1993, 98, 1372-1377.

(87) Perdew, J. P.; Burke, K.; Ernzerhof, M. Generalized Gradient Approximation Made Simple. Phys. Rev. Lett. 1996, 77, 3865-3868.

(88) Keal, T. W.; Tozer, D. J. A semiempirical generalized gradient approximation exchange-correlation functional. J. Chem. Phys. 2004, 121, 5654-5660.

(89) Hirsch, A.; Chen, Z.; Jiao, H. Spherical Aromaticity in $\mathrm{I}_{h}$ Symmetrical Fullerenes: The $2(N+1)^{2}$ Rule. Angew. Chem., Int. Ed. 2000, 39, 3915-3917.

(90) Bühl, M.; Hirsch, A. Spherical aromaticity of fullerenes. Chem. Rev. 2001, 101, 1153-1183.

(91) Reiher, M.; Hirsch, A. From Rare Gas Atoms to Fullerenes: Spherical Aromaticity Studied From the Point of View of Atomic Structure Theory. Chem. - Eur. J. 2003, 9, 5442-5452.

(92) Johansson, M. P.; Sundholm, D.; Vaara, J. $A_{32}$ : A 24-carat golden fullerene. Angew. Chem., Int. Ed. 2004, 43, 2678-2681.

(93) Chen, Z.; King, R. B. Spherical aromaticity: Recent work on fullerenes, polyhedral boranes, and related structures. Chem. Rev. 2005, 105, 3613-3642.

(94) Johansson, M. P.; Jusélius, J.; Sundholm, D. Sphere Currents of Buckminsterfullerene. Angew. Chem., Int. Ed. 2005, 44, 1843-1846.

(95) Johansson, M. P. On the Strong Ring Currents in $B_{20}$ and Neighboring Boron Toroids. J. Phys. Chem. C 2009, 113, 524-530.

(96) Taubert, S.; Sundholm, D.; Pichierri, F. Magnetically Induced Currents in [n]Cycloparaphenylenes, $\mathrm{n}=6-11$. J. Org. Chem. 2010, 75, $5867-5874$.

(97) Sundholm, D. $\mathrm{C}_{72}$ : Gaudiene a Hollow and Aromatic AllCarbon Molecule. Phys. Chem. Chem. Phys. 2013, 15, 9025-9028.

(98) Rauhalahti, M.; Muñoz-Castro, A.; Sundholm, D. Magnetic response properties of gaudiene - a cavernous and aromatic carbocage. Phys. Chem. Chem. Phys. 2016, 18, 18880-18886.

(99) Ahrens, J.; Geveci, B.; Law, C. ParaView: An End-User Tool for Large Data Visualization, Visualization Handbook; Elsevier, 2005, ISBN-13: 978-0123875822, see also: http://www.paraview.org.

(100) gnuplot homepage, http://www.gnuplot.info/.

(101) Humphrey, W.; Dalke, A.; Schulten, K. VMD: Visual molecular dynamics. J. Mol. Graphics 1996, 14, 33-38.
(102) gimp: GNU Image Manipulation Program, http://www.gimp. org.

(103) Iijima, S.; Brabec, C.; Maiti, A.; Bernholc, J. Structural flexibility of carbon nanotubes. J. Chem. Phys. 1996, 104, 2089-2092.

(104) Liu, L.; Jayanthi, C. S.; Wu, S. Y. Structural and electronic properties of a carbon nanotorus: Effects of delocalized and localized deformations. Phys. Rev. B 2001, 64, No. 033412.

(105) Ormsby, J. L.; King, B. T. Clar Valence Bond Representation of $\pi$-Bonding in Carbon Nanotubes. J. Org. Chem. 2004, 69, 42874291.

(106) Yang, L.; Anantram, M. P.; Han, J.; Lu, J. P. Band-gap change of carbon nanotubes: Effect of small uniaxial and torsional strain. Phys. Rev. B 1999, 60, 13874-13878.

(107) Lu, X.; Chen, Z. Curved Pi-Conjugation, Aromaticity, and the Related Chemistry of Small Fullerenes $\left(\mathrm{C}_{60}\right)$ and Single-Walled Carbon Nanotubes. Chem. Rev. 2005, 105, 3643-3696.

(108) Laird, E. A.; Kuemmeth, F.; Steele, G. A.; Grove-Rasmussen, K.; Nygård, J.; Flensberg, K.; Kouwenhoven, L. P. Quantum transport in carbon nanotubes. Rev. Mod. Phys. 2015, 87, 703-764.

(109) Valiev, R. R.; Fliegl, H.; Sundholm, D. Closed-shell paramagnetic porphyrinoids. Chem. Commun. 2017, 53, 9866-9869.

(110) Valiev, R. R.; Fliegl, H.; Sundholm, D. Optical and magnetic properties of antiaromatic porphyrinoids. Phys. Chem. Chem. Phys. 2017, 19, 25979-25988.

(111) Valiev, R. R.; Benkyi, I.; Konyshev, Y. V.; Fliegl, H.; Sundholm, D. Computational Studies of Aromatic and Photophysical Properties of Expanded Porphyrins. J. Phys. Chem. A 2018, 122, 4756-4767.

(112) Valiev, R. R.; Baryshnikov, G. V.; Sundholm, D. Relations between the aromaticity and magnetic dipole transitions in the electronic spectra of hetero[8]circulenes. Phys. Chem. Chem. Phys. 2018, 20, 30239-30246. 